\title{
Anti-Reflecting Absorbing Layers for Electrochemical and Biophotonic Applications
}

\author{
Ausserre $D^{1 *}$, Abou Khachfe $\mathbf{R}^{2}$, Roussille $\mathrm{L}^{1}$, Brotons $\mathbf{G}^{1}$, Vonna $\mathrm{L}^{3}$, Lemarchand $\mathrm{F}^{4}$, Zerrad $\mathbf{M}^{4}$ and $A m r a C^{4}$ \\ ${ }^{1}$ Molecular Landscapes Laboratory, UMR CNRS 6283, Université du Maine, BP 535, 72085 Le Mans Cedex 9, France \\ ${ }^{2} A U L$ University, South street, Jadra, Lebanon \\ ${ }^{3}$ Laurent VONNA, Faculté des Sciences et Techniques - Université de Haute Alsace, Institut de Science des Matériaux de Mulhouse, 15, rue Jean Starcky - BP 2488, \\ 68057 Mulhouse Cedex, France \\ ${ }^{4}$ Institut Fresnel, Group Concept, UMR CNRS 7249, Faculté des Sciences et Techniques de St Jérôme, 13397 Marseille Cedex 20 , France
}

\begin{abstract}
Anti-reflecting layers deposited on flat surfaces make molecular films visible in reflecting light microscopy. For centuries, single Anti-Reflecting layers have been implicitly associated with dielectric materials. We recently demonstrated that anti-reflecting layers could be achieved out of absorbing materials such as metals as well, but only when used as backside layers where illumination and detection are performed through a supporting window. Fortunately, this corresponds to the best geometry when envisaging biophotonic or electrochemical applications at the solid/liquid interface. Here we explain how single absorbing anti-reflecting layers can serve each of these applications, and both simultaneously.
\end{abstract}

Keywords: Anti-Reflecting; Electrochemical; Biophotonic applications; Zeolites

\section{Introduction}

Electrochemical processes often take place at the solid/liquid interfaces as it the case for many other physical and chemical reactions, for instance dissolution, crystallization, adsorption, polymerization or covalent grafting. For this reason, the most efficient electrochemical and surface based devices often involve a high solid/liquid specific area (porous electrodes for batteries, zeolites and microporous clusters for catalysis, ball columns for Liquid Chromatography). Electro-chemical processes at interfaces are of particular interest for major current concerns such as clean energy production and storage, actuation in very large scale integrated systems, chemical and biosensors, catalysts, surface reactivity, neural activity or enzymatic reactions. They are complex and multi-step phenomena, especially difficult to understand and to control in applications. In order to make things simpler, the research done in this area mainly lies on experiments conducted on flat model surfaces. As a consequence, most of experimental tools used in this domain are surface techniques. With progressive integration of complex analytical devices, the localized character of electrochemical phenomena is of increasing interest. Also, real time reaction kinetics give essential information about the mechanisms. For these two reasons, there is a growing need for combined electrochemical and in situ imaging tools [1]. The simplest imaging techniques are optical ones. Unfortunately, electrochemical processes and measurements require to set in the solution at least one counter electrode (CE) in regard of the investigated surface (working electrode, WE). Since optical imaging requires a free path for light to the surface, it is difficult to combine electrochemical and optical techniques on the same side of the sample. On the other hand, the solid surface must be conductive and it is well known that most conductive materials are strongly absorbing visible light, so that observation trough the electrode seems impossible. Hence it is an accepted idea that the only way to combine optical imaging and electrochemical techniques is to make them coexist in the same half-space. The present paper aims to shake this idea in proposing a way to combine the conductive character of the layers, which is essential when using them as electrodes, with transparency and high contrast optical imaging of the electrode/electrolyte interface.
It is based on the use ultrathin Anti-Reflecting Absorbing (ARA) layers [2] observed in reflected light with an inverted optical microscope. The high sensitivity comes from the background extinction associated with the AR character. Beside real time in situ imaging with subnanometer sensitivity, the main advantage of this technique is to leave open the half-space needed to implement the fluid cell and the electrodes. Its main limitation is to be restricted to ultrathin layers in the case of strongly absorbing materials such as metals. This excludes some applications, for instance corrosion monitoring.

\section{AR absorbing layers}

As usual single AR layers, ARA layers are defined by two conditions, one on their refractive index and one on their thickness. Writing these conditions in terms of the complex dielectric constant $\varepsilon_{1}=\varepsilon_{1}{ }^{\prime}-j \varepsilon_{1}$ of the layer $\varepsilon_{1}$ ' and $\varepsilon_{1}$ " being real quantities, and supposing a highly absorbing layer material, we get [1]:

$$
\begin{aligned}
& \varepsilon_{1}^{\prime}=\sqrt{\varepsilon_{0} \varepsilon_{3}} \\
& e_{1}=\frac{\lambda}{2 \pi} \frac{\left(\sqrt{\varepsilon_{0}}-\sqrt{\varepsilon_{3}}\right)}{\varepsilon_{1}^{\prime \prime}}
\end{aligned}
$$

where $e_{1}$ is the physical layer thickness, $\varepsilon_{0}$ and $\varepsilon_{3}$ are the dielectric constants of, respectively, the incident and emergent materials, supposed purely dielectric, and $\lambda$ is the wavelength of light. Subscript 2 is booked for an additional object to be inserted between materials 1 and 3. The index condition given by Equation (1) is exact within a fraction of a percent for all layer materials, whatever their absorption. It merges with the well-known equation $\boldsymbol{n}_{1}{ }^{2}=\boldsymbol{n}_{0} \boldsymbol{n}_{3}$ for dielectrics

*Corresponding author: Ausserre D, Molecular Landscapes Laboratory, UMR CNRS 6283, Université du Maine, BP 535, 72085 Le Mans Cedex 9, France, Tel: +33 6082848 00; E-mail: ausserre@univ-lemans.fr

Received June 03, 2014; Accepted July 14, 2014; Published July 21, 2014

Citation: Ausserre D, Abou Khachfe R, Roussille L, Brotons G, Vonna L, et al (2014) Anti-Reflecting Absorbing Layers for Electrochemical and Biophotonic Applications. J Nanomed Nanotechnol 5: 214. doi: 10.4172/2157-7439.1000214

Copyright: @ 2014 Ausserre D, et al. This is an open-access article distributed under the terms of the Creative Commons Attribution License, which permits unrestricted use, distribution, and reproduction in any medium, provided the original author and source are credited. 
Citation: Ausserre D, Abou Khachfe R, Roussille L, Brotons G, Vonna L, et al. (2014) Anti-Reflecting Absorbing Layers for Electrochemical and Biophotonic Applications. J Nanomed Nanotechnol 5: 214. doi: 10.4172/2157-7439.1000214

Page 2 of 5

when the layer absorption vanishes. Equation (2), which assumes highly absorbing materials, tells us essential features. The first one is that the refractive index $n_{0}=\sqrt{ } \varepsilon_{0}$ of the incident medium (where the light comes from) must be larger than the refractive index $n_{3}=\sqrt{\varepsilon_{3}}$ of the emergent medium (where the light goes after passing through the layer). Otherwise, the thickness $\mathrm{e}_{1}$ of the intermediate layer as given by Equation (2) would be negative. This is a striking illustration of the asymmetry of the reflectivity when absorbing layers are added to interfaces (as in way mirrors). The second one is that $\mathrm{e}_{1}$ is very small. It is counted in nanometers. This is fortunate since the anti-reflecting condition takes its origin in the destructive interference between the light amplitudes reflected by the front and the back surfaces of the absorbing layer. Would the layer be thicker, the light would be completely absorbed by the layer material before reaching the rear surface and the interference could not occur [3].

With metals, Equation (1) is barely satisfied since normally $\varepsilon_{1}^{\prime}$ is negative. However, even if non zero, the layer reflectivity finds a minimum at a layer thickness which is close to that given by Equation (2), and the sensitivity of the backside layer in imaging applications is yet excellent. We name Near ARA layers (NARA) such layers made of an arbitrary absorbing material which does not conform to Equation (1), but with optimized thickness. Since dielectric properties of metals are strongly dependant of wavelength, it is also possible to better approach Equation (1) by selecting the working wavelength. We illustrate the potential of NARA layers by considering the example of gold, which is an important material for electrochemistry and biophotonics. Assuming that the optical properties of ultrathin gold films are unchanged with respect to the bulk, we characterize the sensitivity of the layer by estimating the contrast $\mathrm{C}=\left(\mathrm{I}_{\text {spot }}-\mathrm{I}_{\text {background }}\right) /\left(\mathrm{I}_{\text {spot }}+\mathrm{I}_{\text {background }}\right)$ of a dielectric spot with refractive index 1.46 lying on it. Such a refractive index is typical of an organic Self-Assembled Monolayer (SAM). In Figure 1, the expected contrast of this object, when observed in water, is shown for various wavelengths as a function of the object thickness. The object is labeled 2. The value of the gold layer thickness was chosen to be $3 \mathrm{~nm}$, which agrees reasonably well with Equation (2). The contrast of small objects appears negative and the wavelength giving the higher contrast is 488 $\mathrm{nm}$ (red curve). For object thicknesses less than $20 \mathrm{~nm}$, the contrast is a linear function of the spot thickness with a slope of about $-4 \%$ per nm. Assuming that detecting a contrast of $0.04 \%$ is a common performance, the NARA gold layer should allow imaging a layer less than $10 \mathrm{pm}$ thick. With layers made of optimal materials obeying Equation (1), the real time sensitivity would become better than a picometer. It should be understood that such a thickness is an average over molecules dispersed over the surface, and separated two by two by a distance smaller than the optical resolution of the microscope.

To resume, ultrathin layers of strongly absorbing materials may form anti-reflecting layers and allow high contrast imaging, but only when they are used as backside layers of a solid window. Fortunately, this configuration is precisely the one suited in two major domains of application: electrochemistry and biophotonics. In the following, we sketch perspectives for these two applications.

\section{Electrochemistry}

We consider glucose electrochemical sensors as an example, because monitoring the glucose amount in blood is an important issue and current monitoring tools are mostly electrochemical ones. Examples are given in Figure 2. They were reproduced from reference [4] (Ask for authorization).

The left part of the figure displays the exploded view of the Freestyle glucose sensor commercialized by the ABBOTT company. Like most electrochemical devices, it includes a fluid cell with three

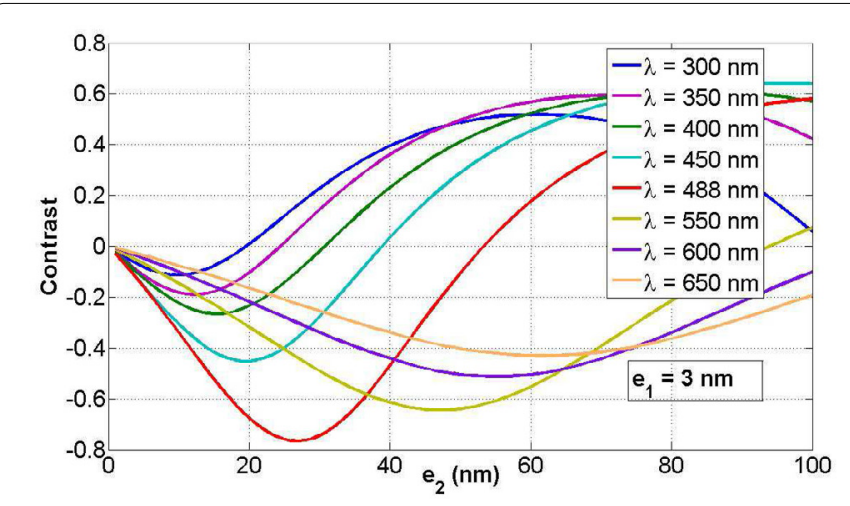

Figure 1: Optical contrast of a spot with refractive index 1.46 grafted on a $3 \mathrm{~nm}$ thick gold layer, as a function of the spot thickness, for various visible wavelengths.
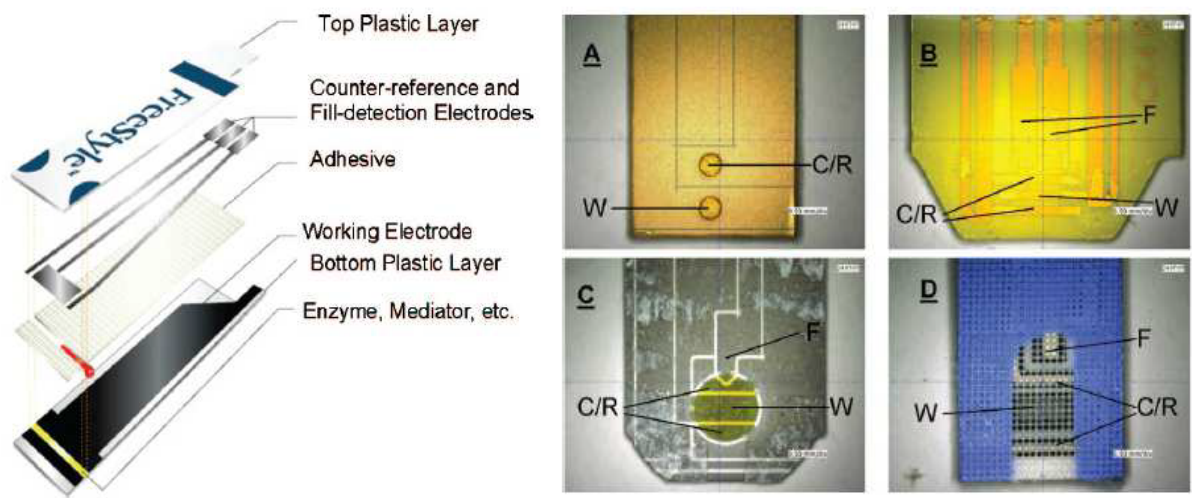

Figure 2: Examples of commercial glucose sensors (taken from Ref. 4 with author permission). 
Citation: Ausserre D, Abou Khachfe R, Roussille L, Brotons G, Vonna L, et al. (2014) Anti-Reflecting Absorbing Layers for Electrochemical and Biophotonic Applications. J Nanomed Nanotechnol 5: 214. doi: 10.4172/2157-7439.1000214

Page 3 of 5

measuring electrodes, the fourth one being added for the control of correct filling. The blood is inserted in the test strip (partially red in the figure), typically several millimeters large, and the electric signal is generated from RedOx reactions occurring at the flat working electrode (WE). In this peculiar example, the WE is made of carbon. The right part of the Figure shows other examples with WEs made of other materials, such as Nickel or gold. Thanks to selective reduction of the glucose with a specific enzyme, and transport of the gained electrons via specific mediators to the $\mathrm{WE}$, the chemical reaction occurring at the WE surface is not supposed to evolve with device use. However, other non-specific RedOx reactions competing with this process, or non-specific adsorption, may modify the WE so that in practice the reliability of the measurement may be altered. In the development of such systems, it would clearly be useful to observe in situ the surface of the WE. At the level of principles, Figure 3a illustrates how the ARA (or NARA) layers make it possible. In the instrument sketched in the figure, that we may name ElectroChemical Optical Microscope (ECOM), the window is separating the space in two halves, one for the reflection microscopy and one for the fluid cell and the electrochemistry. The focus of the microscope is made on the interface between the liquid and the electrode covering the window. In many electrochemical sensors, chemical and physical modifications of the WE surface are part of the working system, and important to characterize. In other applications, such as electrochemical deposition, modification of the surface electrode is the final goal of the electrochemical process. Figure $3 \mathrm{~b}$ presents a high magnification image of copper grains nucleating on a gold working electrode in the early stage of an electrochemical transfer, obtained using the setup displayed in Figure 3a. The lateral resolution appears much better than one micron.

The combination of electrochemical and optical tools in a single instrument provides a unique monitoring tool. In other applications, such as anti-corrosion layers, the electrode integrity must be monitored, and the technique we describe here could help to detect early damage. In most research projects at least, ranging from catalysis and analytical chemistry to electrochemical screen printing, FET sensors or solar cells, it is a key point to understand what happens at the surface of an electrode, and coupling high resolution optical imaging and local or averaged electrochemical measurements should bring essential information.

\section{Biophotonics}

Biophotonic assays on surfaces aim to probe ligand-analyte interactions involving biomolecules, for instance antigen and antibody. The ligands are grafted on the surface and form a bioactive layer intended to selectively capture the complementary analytes. In biochips, the bioactive layer is distributed into separate spots corresponding to different ligands. Differentiating the full and empty spots after incubation requires enough sensitivity to detect, and preferentially measure the very small amount of additional material which is present on positive spots, resulting from the capture of the analytes. When the detection method is label free, great care must be taken in order to reduce non specific interactions [5], and this demands the strict respect of suited biochemistry protocols, which consist in practice in making a given sequence of solutions flow over the surface at a given rate. Such protocols are barely compatible with drying and rehydrating the surface. Therefore, the detection technique must be able to operate in situ in a fluid cell. Moreover, in order to evaluate the strength of the ligand-analyte affinity, it is necessary to probe capture kinetics, which requires a transduction (detection) technique operating in realtime. The most wide-spread and versatile technique to do that is Surface Plasmon Resonance (SPR) [6]. In order to get the optical resonance, a thin metal layer is deposited against a solid surface and supports the bioactive layer. For various reasons including good chemical inertia in water, gold is the metal which is commonly used.

In order to compare the performances of NARA layers with SPR, we had to choose gold as the layer material. It was deposited on the backside of a glass window and used as illustrated in Figure 4a. Although the layer thickness was slightly larger than suited (5 $\mathrm{nm}$ ), real time sub-nanometer sensitivity could easily be achieved in

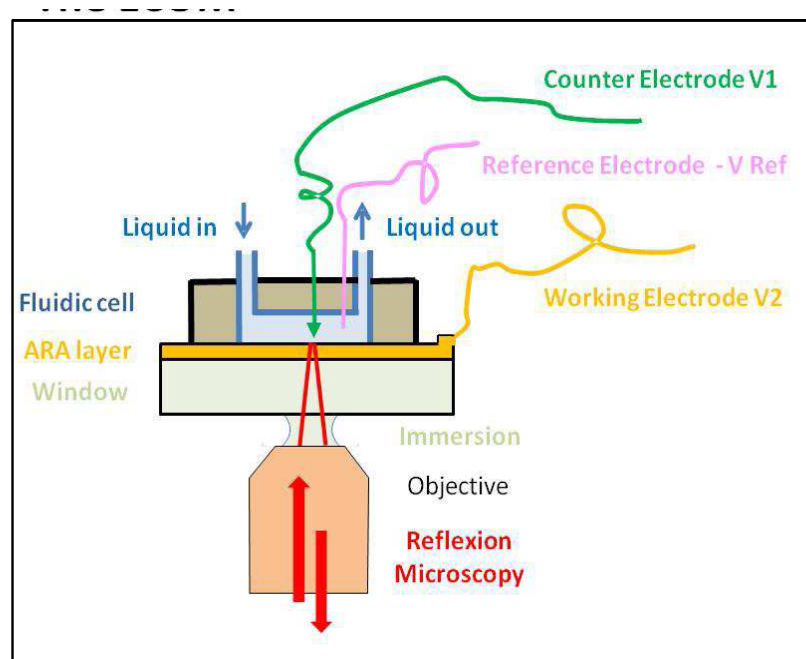

a

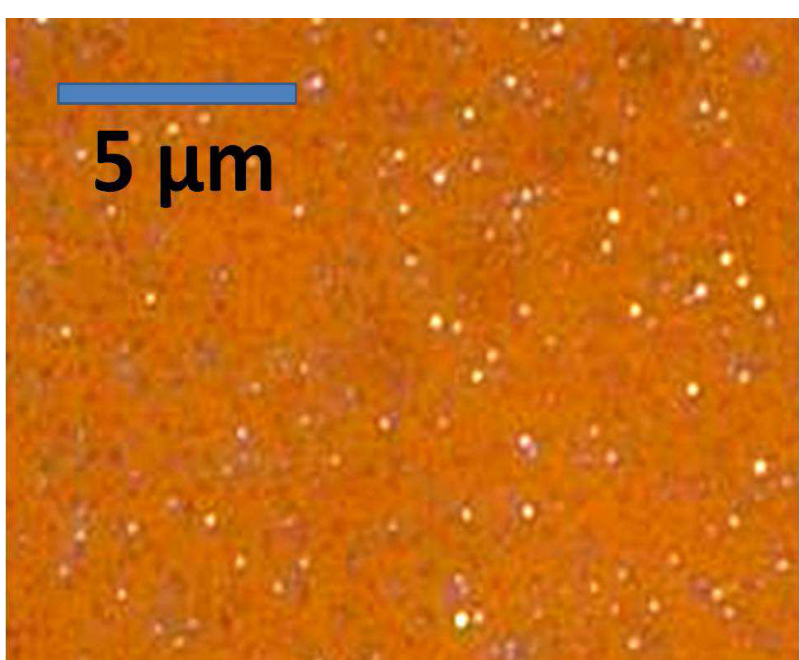

b

Figure 3: a) Principle of the wide field ElectroChemical Optical Microscope (ECOM). The presence of the ARA layer brings the sensitivity required for probing local electrochemical phenomena at a molecular level. Simultaneously, the upper space is used for controlling and measuring electrochemical processes. This apparatus will allow to link electrochemical measurements and related physical changes at the electrode surface. b) First stage of the electrochemical transfer of copper on gold using a two electrodes set up; V1 - V2 $=200 \mathrm{mV}$; The V1 electrode is located in the upper right region of the sample, $500 \mu \mathrm{m}$ away from the zone represented in the image. The distance of this electrode to the surface is also about $500 \mu \mathrm{m}$. 


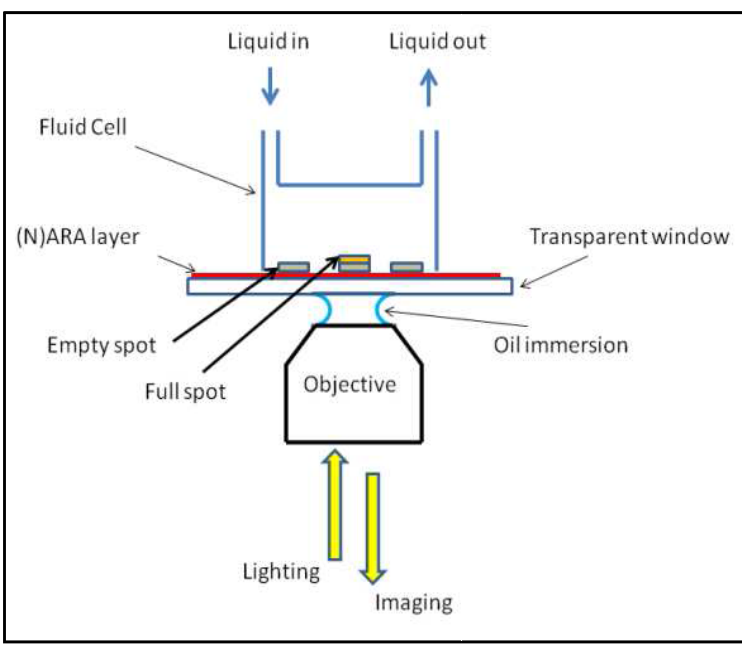

a

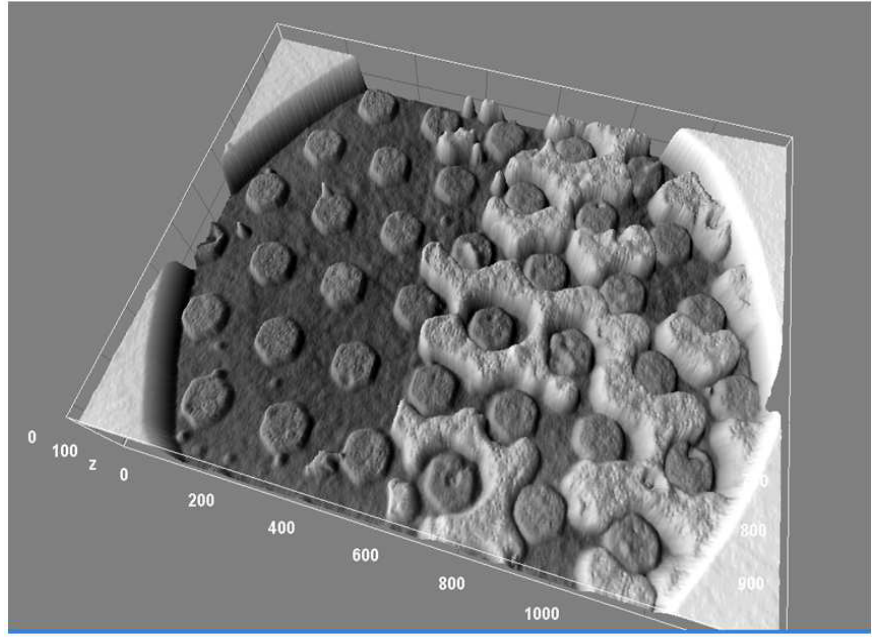

b

Figure 4: Non specific adsorption of BSA on a patterned gold surface in a fluidic cell. a) Schematic view of the setup. The fluidic cell is sealed by a glass window supporting identical biochip spots. In immobilization or affinity measurement experiment, the capture of the ligands attaching to the spots increases the optical thickness of the spot, which produces a change in the reflected light intensity. The method is widefield, all the spots are visualized at once. b) $3 \mathrm{D}$ reconstruction of an image obtained using the setup of Figure a. What represents this image is explained in the text. Numbers are arbitrary marks.

water. The aperture of the microscope was about 30 degrees. A $3 \%$ contrast of the empty spots (before capture events) is expected when working in these conditions, and this is roughly what we obtained. An extensive study will be reported elsewhere. Our present goal is just to illustrate the possibilities of our approach. Figure $2 b$ is a topographic view of a typical image of a non homogeneous surface as captured in the microscope. It is decorated with spots of alkanethiols monolayers chemically grafted on the surface using a microcontact printing technique [7], and following a procedure described earlier [8]. Each of these spots is $16 \mu \mathrm{m}$ large and $1.6 \mathrm{~nm}$ thick and they globally form a 2D-hexagonal periodic pattern. The sample is also used as the window of a fluidic cell where Bovine Serum Albumin (BSA) flows according to conditions where non-specific adsorption of BSA takes place on the bare gold surface and not on the spots [6]. The image shown in the figure was captured in situ during that process. The grey scale was inverted because the contrast is negative as it was previously mentioned (Figure 1). Beside the outside circle which is the mark of the field aperture, the image is divided in two contrasted parts (left and right). The frontier between them corresponds to the limit of the PDMS fluid cell. Water could flow underneath the PDMS wall because it was insufficiently tight against the surface, but BSA molecules could not. They only went in the canal over the right zone of the surface. In this region, they are in the course of building a continuous layer surrounding the spots. In the left part of the figure, there are only spots and water. We said previously that for such ultrathin organic films the light intensity in the image is proportional to the optical layer thickness of the film. Knowing the thickness of the spots, that we measured using an AFM prior to the experiment, we could determine the thickness of the BSA layer.

We found it was $4 \mathrm{~nm}$, which corresponds to a known dimension of the molecule [9]. These results illustrate how our technique can be used to probe saturated amounts of analytes for antigen-antibody diagnosis. Since observations are made in real time into the fluid cell, it can be used as well to probe adsorption and immobilization kinetics and thus to measure biomolecular affinities [10].

\section{Conclusion}

ARA and NARA layers bring new possibilities for imaging molecular and sub-molecular layers on conducting surfaces. They offer a wide field imaging alternative to Surface Plasmon Resonance in biophotonic applications, and they allow non-destructive in situ investigation of working electrodes in electrochemistry. Moreover, the development of portable electrochemical biosensors for health, food, water, and environment monitoring and for point of care diagnostics, which is at the crossroads between bioactive surfaces and electrochemical sensors, might benefit from the combination of the two applications highlighted in this paper. At least, one may recall that a major difficulty in the development of label-free biosensors is to make the capture event specific [5]. Electrochemical addressing, where the biocapture is triggered by an electric field [11], may reduce unwanted non-specific deposits, so that optical detection and electrochemical functions could combine as complementary parts of a single device.

\section{Acknowledgement}

This Work was supported by Agence Nationale de la Recherche (grant ANR PNANO07-050)

\section{References}

1. Tao FF, Salmeron M (2011) In situ studies of chemistry and structure of materials in reactive environments. Science 331: 171-174.

2. Ausserré D, Khachfe RA, Amra C, Zerrad M (2014) Absorbing Backside Antireflecting Layers for high contrast imaging in fluid cells.

3. Kats MA, Blanchard R, Genevet P, Capasso F (2013) Nanometre optical coatings based on strong interference effects in highly absorbing media. Nat Mater 12: 20-24

4. Heller A, Feldman B (2008) Electrochemical glucose sensors and their applications in diabetes management. Chem Rev 108: 2482-2505.

5. Silin V, Weetall H, Vanderah DJ (1997) SPR Studies of the Nonspecific Adsorption Kinetics of Human IgG and BSA on Gold Surfaces Modified by SelfAssembled Monolayers (SAMs). Journal of Colloid and Interface Science 185 94-103. 
Citation: Ausserre D, Abou Khachfe R, Roussille L, Brotons G, Vonna L, et al. (2014) Anti-Reflecting Absorbing Layers for Electrochemical and Biophotonic Applications. J Nanomed Nanotechnol 5: 214. doi: 10.4172/2157-7439.1000214

Page 5 of 5

6. Roussille L, Brotons G, Ballut L, Louarn G, Ausserré D, et al. (2011) Surface characterization and efficiency of a matrix-free and flat carboxylated gold sensor chip for surface plasmon resonance (SPR). Anal Bioanal Chem 401: 1601-1617.

7. Mrksich M, Whitesides GM (1995) Patterning self-assembled monolayers using microcontact printing - a new technology for biosensors. Trends in Biotechnology 13: 228-235.

8. Vonna L, Haidara H (2013) Morphology and stability of water condensation structures on a series of self-similar micropatterned substrates. Soft Matter 9: 765-771.
9. Peters Jr (1985) Advances in Protein Chemistry: Serum Album, Academic Press, New York.

10. Long SD, Myszka DG (2006) Handbook of Affinity Chromatography. In: Hage DS (ed). Taylor and Francis; New York.

11. Corgier BP, Bellon S, Anger-Leroy M, Blum LJ, Marquette CA (2009) ProteinDiazonium Adduct Direct Electrografting onto SPRi-Biochip. Langmuir 25: 9619-9623.
Citation: Ausserre D, Abou Khachfe R, Roussille L, Brotons G, Vonna L, et al. (2014) Anti-Reflecting Absorbing Layers for Electrochemical and Biophotonic Applications. J Nanomed Nanotechnol 5: 214. doi: 10.4172/2157-7439.1000214
Submit your next manuscript and get advantages of OMICS Group submissions

Unique features:

- User friendly/feasible website-translation of your paper to 50 world's leading languages Audio Version of published paper

- Digital articles to share and explore

Special features:

- 350 Open Access Journals

- 30,000 editorial team

21 days rapid review process

Quality and quick editorial, review and publication processing

- Indexing at PubMed (partial), Scopus, EBSCO, Index Copernicus and Google Scholar etc

- Sharing Option: Social Networking Enabled

- Authors, Reviewers and Editors rewarded with online Scientific Credits

- Befter discount for your subsequent articles

Submit your manuscript at: www.editorialmanager.com/biochem 